\title{
Measurement of differential renal function in the presence of a pelvic kidney
}

\author{
Aditya Ham • Andre Dobbeleir
}

Received: 19 August 2011 / Accepted: 22 August 2011 /Published online: 17 September 2011

(C) Springer-Verlag 2011

Dear Sir,

In the revised EANM guidelines on ${ }^{99 \mathrm{~m}} \mathrm{Tc}$ DMSA scintigraphy in children [1], it is stated that in the presence of a pelvic kidney the calculated value of relative renal function is inaccurate even when calculated using the geometric mean because of the additional attenuation due to pelvic bone.

We introduced a small modification to the conventional geometric mean method involving the addition of the measurement of a standard. It is then possible to estimate, and hence to correct for, the attenuation due to pelvic bone and at the same time to correct for any difference in body thickness between the hypochondrial and pelvic areas.

The standard is created using an ordinary $60-\mathrm{ml}$ syringe filled with $10 \mathrm{ml}$ of water containing a small amount of radioactivity. The piston of the syringe is pulled until the "active" syringe has more or less the same length as the kidney. Then, three pairs of anterior-posterior images are acquired, the first with only the kidneys in the field of view, the second with the kidneys imaged together with the standard placed such that it is projected at the same place as the right kidney, and the third with the same standard placed such that it is at the same level as the left kidney. By subtracting the first from the second acquisition for each projection, the activity in the standard placed at the level of the right kidney can be determined both for the anterior and the posterior projection, allowing calculation of its geometric mean. The same can be done for the standard placed at the level of the left kidney using the first and the third acquisitions.

As the same standard is used for the right and for the left kidney, if the attenuation is equal, the ratio of the geometric mean of the standard at these two locations is one. The ratio of the calculated geometric mean will differ from unity because of bone attenuation and differences in body thickness at the levels of the right and the left kidney. This calculated value can then be used to correct the geometric mean of the differential renal function calculated from the first data acquisition. The same approach can be used in renography to estimate renal split function in the presence of a pelvic kidney.

\section{Reference}

1. Piepsz A, Colarinha P, Gordon I, Hahn K, Olivier P, Roca I, et al. Guidelines on $99 \mathrm{mTc}-\mathrm{DMSA}$ scintigraphy in children. European Association of Nuclear Medicine; 2009. http://www.eanm.org/ scientific_info/guidelines/gl_paed_dmsa_scin.pdf. Accessed 5 Sep 2011.

\footnotetext{
A. Ham $(\bowtie) \cdot$ A. Dobbeleir

Department of Nuclear Medicine, UZ Gent,

Ghent, Belgium

e-mail: ham.aditya@gmail.com
} 